\title{
Research Square \\ Consistent description of microscopic phenomena by macroscopic observers in quantum theory
}

\section{Alexey Kryukov ( $\nabla$ kryukov@uwm.edu )}

University of Wisconsin, Milwaukee

\section{Research Article}

Keywords: BGS-conjecture, Wigner random matrix theory, measurement problem

Posted Date: May 12th, 2021

DOI: https://doi.org/10.21203/rs.3.rs-501056/v1

License: (c) (i) This work is licensed under a Creative Commons Attribution 4.0 International License. Read Full License 


\title{
Consistent description of microscopic phenomena by macroscopic observers in quantum theory
}

\author{
Alexey A. Kryukov \\ Department of Mathematics \& Natural Sciences, University of Wisconsin-Milwaukee, USA \\ E-mail: kryukov@uwm.edu
}

\begin{abstract}
Quantum mechanics is the foundation of modern physics that is thought to be applicable to all physical phenomena, at least in principle. However, when applied to macroscopic bodies, the theory seems to be inconsistent. Wigner's friend and related thought experiments demonstrate that accounts by different observers described by the rules of quantum mechanics may be contradictory. Although still highly debated, such experiments seem to demonstrate an incompatibility of quantum mechanics with the usual rules of logic. Alternatively, one of the hidden assumptions in the thought experiments must be wrong. For instance, the argument is invalidated if macroscopic observers cannot be considered as physical systems described by the rules of quantum theory. Here we prove that there is a way to apply the rules of quantum mechanics to macroscopic observers while avoiding contradictory accounts of measurement by the observers. The key to this is the random noise that is ever present in nature and that represents the uncontrollable part of interaction between measured system and the surroundings in classical and quantum physics. By exploring the effect of the noise on microscopic and macroscopic bodies, we demonstrate that accounts of Wigner, the friend and other agents all become consistent. Our result suggests that the existing attempts to modify the Schrödinger equation to account for measurement results may be misguided. More broadly, the proposed mechanism for modeling measurements underlies the phenomenon of decoherence and is shown to be sufficient to explain the transition to Newtonian physics in quantum theory.
\end{abstract}

\section{Introduction}

Applicability of quantum mechanics to macroscopic bodies has been the theme of fierce debates since the inception of quantum theory. EPR, Schrödinger cat, Wigner's friend and numerous other thought experiments were proposed to demonstrate that the rules of quantum mechanics do not agree with the macroscopic reality that we know and can even result in contradictory accounts of reality by different observers.

At the same time, the quantum mechanics is experimentally confirmed and is certainly true for microscopic systems. Furthermore, quantum mechanics is also a basis for our understanding of important cosmological processes such as the black hole radiation, the mass-radius relation of white dwarf stars and the birth of the universe. So it seems to be applicable in principle to the world of small and big alike. Hence, the paradox that has yet to be resolved.

Here we show a possible way out of the conundrum by demonstrating how the state of a macroscopic body can satisfy the Schrödinger equation while the body behaves in the way consistent with everyday observations. We combine a recently discovered tight geometrybased relationship of the Schrödinger and Newtonian dynamics with the theory of a classical measurement and Wigner's random matrix theory to derive the dynamics of the quantum state 
of a microscopic system under an ideal measurement. What follows is an agreement between all macroscopic observers about the results of a measurement on the system, and, as a consequence, a consistent view of microscopic and macroscopic reality by the observers.

\section{Results}

It has been recently established that the Schrödinger and Newtonian dynamics are related in a more intimate than previously thought possible way. Namely, the Newtonian dynamics of a system was identified with the Schrödinger dynamics with the state of the system constrained to a submanifold in the space of states $[1,2,3]$. This is analogous to the dynamics of a classical mechanical system with a constraint, for example, a bead on a wire. However, this time the dynamics is determined by the Schrödinger equation and the constraint is imposed on the state function in the Hilbert space of states.

In the case of a single spinless particle, the transition to Newtonian dynamics happens when the state of the particle in coordinate representation is constrained to the set $M_{3,3}^{\sigma}$ of all functions of the form $\varphi(\mathbf{x})=g_{\mathbf{a}, \sigma}(\mathbf{x}) e^{i \mathbf{p x} / \hbar}$. Here $g_{\mathbf{a}, \sigma}=\left(\frac{1}{2 \pi \sigma^{2}}\right)^{3 / 4} e^{-\frac{(\mathbf{x}-\mathbf{a})^{2}}{4 \sigma^{2}}}$ is the Gaussian function of a sufficiently small variance $2 \sigma^{2}$ centered at a point $\mathbf{a}$ in the Euclidean space $\mathbb{R}^{3}$ and $\mathbf{p}$ is a fixed vector in $\mathbb{R}^{3}$. In quantum mechanics the state $\varphi(\mathbf{x})$ represents a narrow wave packet with group velocity $\mathbf{p} / m$, where $m$ is the mass of the particle. The states in $M_{3,3}^{\sigma}$ are defined up to a physically unobservable constant phase factor $e^{i \alpha}$, i.e., they are identified with the elements $\{\varphi\}$ of the projective space $C P^{L_{2}}$.

The set of all pairs $(\mathbf{a}, \mathbf{p})$ can be identified with the classical phase space $\mathbb{R}^{3} \times \mathbb{R}^{3}$ of possible positions a and momenta $\mathbf{p}$ of a particle. The map $\Omega:(\mathbf{a}, \mathbf{p}) \longrightarrow g_{\mathbf{a}, \sigma} e^{i \mathbf{p x} / \hbar}$ identifies then the classical phase space with the submanifold $M_{3,3}^{\sigma}$ of $C P^{L_{2}}$. The six-dimensional manifold $M_{3,3}^{\sigma}$ is embedded into the space of states in a very special way. Moving along $M_{3,3}^{\sigma}$ corresponds to crossing the dimensions of the Hilbert space of states. There are no vectors in the Hilbert space orthogonal to all of $M_{3,3}^{\sigma}$. Instead, the points of $M_{3,3}^{\sigma}$ represent an overcomplete basis in the Hilbert space [4].

As explained in $[3,5]$ and the Methods, under the Schrödinger evolution of the particle in a potential $V$ with the initial state $\varphi$ in $M_{3,3}^{\sigma}$, the velocity of state at $t=0$ can be decomposed into four physically meaningful orthogonal components. The two components tangent to the classical phase space $M_{3,3}^{\sigma}$ are the usual classical velocity and acceleration of the particle. The other two components orthogonal to $M_{3,3}^{\sigma}$ are the phase velocity and the velocity of spreading of the wave-packet. The norm of the total velocity $d \varphi / d t$ at $t=0$, i.e., the speed of motion of the initial state $\varphi$, is given by the formula

$$
\left\|\frac{d \varphi}{d t}\right\|^{2}=\frac{\bar{E}^{2}}{\hbar^{2}}+\frac{\mathbf{v}^{2}}{4 \sigma^{2}}+\frac{m^{2} \mathbf{w}^{2} \sigma^{2}}{\hbar^{2}}+\frac{\hbar^{2}}{32 \sigma^{4} m^{2}} .
$$

Here $\mathbf{v}=\frac{\mathbf{p}}{m}$ and $\mathbf{w}=-\frac{\nabla V}{m}$ are the classical velocity and acceleration of the particle and $\hbar$ is the Planck constant. The first term is the square of the phase velocity and the last term is the square of the velocity of spreading of the wave packet. When the state is constrained to the manifold $M_{3,3}^{\sigma}$, the only surviving terms are the classical velocity and acceleration and the motion follows the classical Newtonian dynamics in the phase space.

A simpler way to see the transition from Schrödinger to Newtonian dynamics is by applying the variational principle. The variation of the functional

$$
S[\varphi]=\int \bar{\varphi}(\mathbf{x}, t)\left[i \hbar \frac{\partial}{\partial t}-\widehat{h}\right] \varphi(\mathbf{x}, t) d^{3} \mathbf{x} d t
$$


with the Hamiltonian $\widehat{h}=-\frac{\hbar^{2}}{2 m} \Delta+V(\mathbf{x}, t)$ yields the Schrödinger equation for $\varphi$. For the states $\varphi$ constrained to the manifold $M_{3,3}^{\sigma}$, this functional reduces to the classical action

$$
S=\int\left[\mathbf{p} \frac{d \mathbf{a}}{d t}-h(\mathbf{p}, \mathbf{a}, t)\right] d t
$$

where $h(\mathbf{p}, \mathbf{a}, t)=\frac{\mathbf{p}^{2}}{2 m}+V(\mathbf{a}, t)+$ const is the Hamiltonian function for the particle. It follows that the variation of the functional (2) subject to the constraint that $\varphi$ belongs to $M_{3,3}^{\sigma}$ yields Newtonian equations of motion. This result generalizes to an arbitrary system of $n$ particles with the state constrained to the classical phase space submanifold $M_{3 n, 3 n}^{\sigma}=M_{3,3}^{\sigma} \otimes \ldots \otimes M_{3,3}^{\sigma}$ in the space of states of the system. That is, the constrained state is the product of states of the $n$ particles in the system with the state of each particle constrained to a copy of $M_{3,3}^{\sigma}$. A more detailed analysis leads to the following theorem [2]:

(A) The Newtonian dynamics of an arbitrary mechanical system is the Schrödinger dynamics of that system with the state constrained to the classical phase space submanifold of the space of states of the system. Furthermore, the Schrödinger dynamics is the only unitary evolution that reduces to the Newtonian one under the said constraint.

The identification of the classical phase space of a particle with the manifold $M_{3,3}^{\sigma}$ also provides one with a useful metric relationship. Let $M_{3}^{\sigma}$ denote the submanifold of $M_{3,3}^{\sigma}$ of the Gaussian states $g_{\mathbf{a}, \sigma}=\left(\frac{1}{2 \pi \sigma^{2}}\right)^{3 / 4} e^{-\frac{(\mathbf{x}-\mathbf{a})^{2}}{4 \sigma^{2}}}$. The map $\omega: \mathbf{a} \longrightarrow g_{\mathbf{a}, \sigma}, \omega\left(\mathbb{R}^{3}\right)=M_{3}^{\sigma}$ identifies the submanifold $M_{3}^{\sigma}$ of $C P^{L_{2}}$ with the classical space $\mathbb{R}^{3}$. If $\theta\left(g_{\mathbf{a}, \sigma}, g_{\mathbf{b}, \sigma}\right)$ denotes the distance from $g_{\mathbf{a}, \sigma}$ to $g_{\mathbf{b}, \sigma}$ in $M_{3}^{\sigma}$ in the metric on the space of states $C P^{L_{2}}$ and $(\mathbf{a}-\mathbf{b})^{2}$ is the square of the Euclidean distance between the corresponding points $\mathbf{a}$ and $\mathbf{b}$ in $\mathbb{R}^{3}$, then

$$
e^{-\frac{(\mathbf{a}-\mathbf{b})^{2}}{4 \sigma^{2}}}=\cos ^{2} \theta\left(g_{\mathbf{a}, \sigma}, g_{\mathbf{b}, \sigma}\right) \text {. }
$$

Likewise, for arbitrary states $\varphi=g_{\mathbf{a}, \sigma} e^{i \mathbf{p x} / \hbar}$ and $\psi=g_{\mathbf{b}, \sigma} e^{i \mathbf{q} \mathbf{x} / \hbar}$ in the classical phase space $M_{3,3}^{\sigma}$ the distances in the Euclidean phase space and the space of states are related by

$$
e^{-\frac{(\mathbf{a}-\mathbf{b})^{2}}{4 \sigma^{2}}-\frac{(\mathbf{p}-\mathbf{q})^{2}}{\hbar^{2} / \sigma^{2}}}=\cos ^{2} \theta(\varphi, \psi) .
$$

The derivation of (4) and (5) is explained in the Methods.

The relation (4) has an immediate implication onto the form of probability distributions of multivariate random variables with values in the classical space and the space of states. Consider a random variable $\varphi$ with values in the space of states $C P^{L_{2}}$. Since the classical space $M_{3}^{\sigma}$ is a submanifold of $C P^{L_{2}}$, we can restrict $\varphi$ to take values in $M_{3}^{\sigma}$. We then have the following result (see [2] and the Methods):

(B) Suppose that the conditional probability of $\varphi$ given that $\varphi$ is in the classical space is described by the normal distribution. Suppose the unconditional probability $P$ of $\varphi$ depends only on the distance between the initial and the observed state. Then $P$ is given by the Born rule for the probability of transition between states: $P=\left|\left(\varphi, \varphi_{0}\right)\right|^{2}$, where $\varphi_{0}$ is the initial state. The opposite is also true.

So, the normal probability distribution is the Born rule in disguise! Moreover, the embedding of the classical space into the space of states allows us to describe the process of measurement in classical and quantum physics on an equal footing, as we are now going to explain. Although the 
discussion will be limited to measurements of the position of a particle or a system of particles, the method is applicable to measurements of other physical quantities as well.

From the experience we know that a generic classical measurement that avoids systematic errors results in a normal, "bell-shaped" distribution of values of the measured quantity. This is consistent with the central limit theorem that describes in this case the cumulative effect of uncontrollable fluctuations from the mean in a series of measurement outcomes. The fluctuations themselves can be attributed to random kicks exerted on the measured particle by the particles of the measuring device and the surroundings. During the period of a measurement, the measured position of the particle undergoes a Brownian motion and is distributed normally. A specific physical model of the measurement is obtained, for instance, by making the measured particle interact bilinearly with oscillators of a heat bath [6, 7]. A single-particle model of the measurement producing the normal distribution of measurement results is also possible. The chaotic motion of the particle through lattice of hard balls under proper conditions (the so-called Lorentz gas model) provides an example $[8,9]$.

Classical measurement of the position of two or more particles can be approached in a similar way. When the particles don't interact with each other, their positions under a measurement are independent random variables. The same is approximately true when the interaction is weak or when a typical time interval of a measurement is too short for the interaction to have any effect on the motion of particles under the measurement. When the interaction is strong and binds the particles together so that they can be thought of as connected by a weightless rigid rod, the center of mass of the system will undergo a translational Brownian motion. The probability distribution of the position of the center of mass of the system at the time of measurement will be normal. Note that in this case a measurement of the position of one of the particles in the system constrains possible values of the position of other particles. For instance, a measurement of the position of one particle in a pair connected by a rod in one dimension determines the position of the second particle.

In mathematical terms, the process of a classical measurement of the position can be identified with a stochastic process, namely a Wiener process, or a random walk that approximates it. It will be now argued that the following hypothesis provides a consistent extension of the mathematical model of a classical measurement into the quantum domain:

(RM) Suppose that the process of measurement of the position of a macroscopic particle or a system of particles is represented accurately by a random walk on the classical position space resulting in the normal distribution of the position. Then the analogous measurement on the corresponding microscopic system can be represented accurately by a random walk on the space of states. The steps of the random walk satisfy the Schrödinger equation for the system with the Hamiltonian represented by a random matrix from the Gaussian unitary ensemble (GUE). The matrices representing the Hamiltonian at two different times are independent and belong to the same ensemble.

We note here that a random matrix $R$ in GUE can be identified with the sum of a complex matrix $A$ whose entries are independent identically distributed normal random variables and the Hermitian conjugate of $A: R=A+A^{\dagger}$.

Random matrices were originally introduced into quantum mechanics by Wigner [10] in a study of excitation spectra of heavy nuclei. Wigner reasoned that the motion of nucleons within the nucleus is so complex that the Hamiltonian of the system can be modeled by a random matrix from an ensemble that respects symmetries of the system but otherwise contains no additional information.

It was later discovered that correlations in the spectrum of random matrices possess a remarkable universality in being applicable to a large number of quantum systems. That includes nuclear, atomic, molecular, scattering systems, chaotic systems with few degrees of freedom as 
well as complex systems, such as solid state systems and lattice systems in the field theory. What is common and what seems to make the random matrix theory applicable to all these systems is their underlying chaotic character. In fact, based on a wealth of experimental evidence it has been conjectured in [11], and the conjecture has been later referred to as the BGS-conjecture, that all quantum systems whose classical counterpart is chaotic exhibit random matrix statistics.

It is known that the Brownian particle in a medium can be considered a classical chaotic system. For instance, the motion of the particle through an appropriate lattice of round obstacles provides a classical chaotic realization of the Brownian motion. A lively discussion of the stochastic, deterministic chaotic and regular characterization of the Brownian motion can be found in $[12,13,14]$. It follows that the hypothesis $(\mathbf{R M})$ is the consequence of the BGS-conjecture, the possibility to attribute chaotic character to classical measurement and the assumption of independence of the Hamiltonian matrices at different times. Note that the choice of the Gaussian unitary ensemble corresponds to time-irreversible systems [15], which agrees with the nature of observation. We are going to demonstrate now that the hypothesis (RM) provides a consistent approach to the process of observation in the classical and quantum mechanics alike.

Let us prove first that for all dimensions $n$ of the space of states, the probability distribution of the displacement of state under the Schrödinger evolution with Hamiltonian $\widehat{h}$ in GUE is isotropic and homogeneous. That is, for all $n$ and all initial states $\{\varphi\}$ in the space of states $C P^{n}$, the components of the vector $\frac{d \varphi}{d t}=-\frac{i}{\hbar} \widehat{h} \varphi$ along any direction in the tangent space $T_{\{\varphi\}} C P^{n}$ are identically distributed random variables. In particular, the probability of reaching a certain state during the evolution may depend only on the distance between the initial and the final state.

Indeed, because the matrix of $\widehat{h}$ is in GUE, the probability density function $P(\widehat{h})$ of $\widehat{h}$ is invariant with respect to conjugations by unitary transformations. That is, $P\left(U^{-1} \widehat{h} U\right)=P(\widehat{h})$ for all unitary transformations $U$ acting in the Hilbert space of states. Also, for all unitary transformations $U$ that leave $\{\varphi\}$ unchanged and therefore all $U$ that act in the tangent space $T_{\{\varphi\}} C P^{n}$, we have

$$
\left(U^{-1} \widehat{h} U \varphi, v\right)=(\widehat{h} U \varphi, U v)=(\widehat{h} \varphi, U v),
$$

where $v$ is a unit vector in $T_{\{\varphi\}} C P^{n}$. It follows that

$$
P(\widehat{h} \varphi, v)=P(\widehat{h} \varphi, U v),
$$

where $P$ is the probability density of the components of $\widehat{h} \varphi$ in the given directions. By a proper choice of $U$, we can make $U v$ to be an arbitrary unit vector in $T_{\{\varphi\}} C P^{n}$, proving the isotropy.

On the other hand, for all unitary operators $V$ in $H$ and a unit vector $v$ in $T_{\{\varphi\}} C P^{n}$, we have

$$
P(\widehat{h} \varphi, v)=P\left(V^{-1} \widehat{h} V \varphi, v\right)=P(\widehat{h} V \varphi, V v) .
$$

Because $V \varphi$ is an arbitrary state and $V v$ is in the tangent space $T_{\{V \varphi\}} C P^{n}$, we conclude with the help of (6) that the probability density function is independent of the initial state of the system.

Next, a general Schrödinger evolution with Hamiltonian $\widehat{h}$ can be thought of as a sequence of steps connecting the points $\varphi_{t_{0}}, \varphi_{t_{1}}, \ldots$ in the space of states. For small time intervals $\Delta t=t_{k}-t_{k-1}$, the state $\varphi_{t_{N}}$ at time $t_{N}$ is given by the time ordered product

$$
\varphi_{t_{N}}=e^{-\frac{i}{\hbar} \widehat{h}\left(t_{N}\right) \Delta t} e^{-\frac{i}{\hbar} \widehat{h}\left(t_{N-1}\right) \Delta t} \ldots e^{-\frac{i}{\hbar} \widehat{h}\left(t_{1}\right) \Delta t} \varphi_{t_{0}} .
$$

Suppose the evolution of the state $\varphi$ of a particle is constrained to the classical space submanifold $M_{3}^{\sigma}$. The points $\varphi_{t_{0}}, \varphi_{t_{1}}, \ldots$ belong then to the submanifold $M_{3}^{\sigma}$ and the steps can be identified 
with translations in the classical space. In other words, the operator $\widehat{h}\left(t_{k}\right)$ represented by a random matrix in GUE must occur as the generator of translation by a vector $\xi_{k}$ in $\mathbb{R}^{3}$, so that $\widehat{h}\left(t_{k}\right)=\xi_{k} \widehat{\mathbf{p}}$, where $\widehat{\mathbf{p}}$ is the momentum operator. All operators of translation commute with each other and (9) yields the following expression:

$$
\varphi_{t_{N}}(\mathbf{x})=\varphi_{t_{0}}\left(x-\xi_{1} \Delta t-\xi_{2} \Delta t-\ldots-\xi_{N} \Delta t\right) .
$$

That is, the initial state is simply translated by the vector

$$
\mathbf{d}=\sum_{k=1}^{N} \xi_{k} \Delta t
$$

in $\mathbb{R}^{3}$

By the hypothesis (RM), the matrices representing $\widehat{h}\left(t_{k}\right)$ are independent and taken from the same ensemble GUE. Therefore, the steps $\xi_{k}$ are independent and identically normally distributed random vectors. It follows that (11) defines a random walk with Gaussian steps in $\mathbb{R}^{3}$. This is known to result in a normal distribution of the position vector $\mathbf{d}$.

With this, we verified that the Schrödinger evolution with the Hamiltonian that satisfies the hypothesis (RM) fulfills both assumptions of the theorem (B). Therefore,

(C) The Schrödinger evolution with the Hamiltonian represented by a family of random matrices in GUE parametrized by the time $t$ and independent for different values of $t$ yields a model of measurement of the position of a particle in classical and quantum physics. Under the constraint that the random walk that approximates the evolution takes place in the submanifold $M_{3}^{\sigma}$, the conditional probability distribution is the normal distribution of the position vector $\mathbf{d}$ in $\mathbb{R}^{3}$. Without the constraint, the evolution (9) satisfies the Born rule. That is, the unconditional probability to find the initial state $\varphi_{0}$ at a point $\varphi$ under the evolution is equal to $\left|\left(\varphi, \varphi_{0}\right)\right|^{2}$.

We have therefore succeeded in finding a model of measurement that puts measurements of the position of a particle in classical and quantum physics on an equal footing and that is consistent with the observation.

A similar result applies to $n$-particle systems. In this case, the classical space $\mathbb{R}^{3}$ of $n$ particles is identified with the $3 n$-dimensional submanifold $M_{3 n}^{\sigma}=M_{3}^{\sigma} \otimes M_{3}^{\sigma} \otimes \ldots \otimes M_{3}^{\sigma}$ in the space of states of the system. According to (RM), the Hamiltonian of the measured system at any time is represented by a random matrix in GUE with independent matrices at different times. The state of the system undergoes a random walk in the space of states. The positions of all $n$ particles are known when the path of the walk passes through a point of the manifold $M_{3 n}^{\sigma}$. In particular, for a pair of particles in a position-entangled state the positions of both particles are known when the position of one of them is known, as then the path of the walk passes through a point of the manifold $M_{3}^{\sigma} \otimes M_{3}^{\sigma}$. As before, the probability of passing through a particular point is given by the Born rule. Note that the position-entangled state of the pair is simply a superposition of classical states of two particles connected by a rod in $M_{3}^{\sigma}$.

The obtained model of a measurement provides an insight into the boundary between quantum to classical. Consider a particle whose initial state is in $M_{3}^{\sigma}$. From (1) and (4) we know that when the distance in $\mathbb{R}^{3}$ is measured in the units of $2 \sigma$, the classical space $\mathbb{R}^{3}$ is metrically identical to the manifold $M_{3}^{\sigma}$. Suppose the particle is too large for the Brownian motion of the particle in the natural surroundings to be noticeable on this scale. In other words, the motion of state constrained to $M_{3}^{\sigma}$ can be assumed to be trivial. We know that the random nature of the Hamiltonian of the particle in the surroundings results in the direction-independent probability distribution of steps of the random walk in the space of states of the particle. It follows that 
in the absence of an external potential the motion of state of the particle in the space of states will be trivial as well. If an external potential is applied to the particle and the initial state of the particle is in $M_{3}^{\sigma}$, the theorem (A) asserts that the particle will move classically.

As an example, in the Methods we find the displacement of a particle of radius $1 \mathrm{~mm}$ in still air in normal conditions. Assuming the particle's position is measured by scattering and observing visible light, the displacement during the time interval of the measurement is found to be of the order of $10^{-12} \mathrm{~m}$. The corresponding displacement of state in the space of states is of the order of $10^{-7} \mathrm{rad}$. These displacements are too small to be observed in the described experiment. The state of the particle remains "frozen", the particle is constrained to $M_{3}^{\sigma}$ and no superpositions of states of a different position of the particle can be observed.

On the contrary, suppose the particle is sufficiently small, but not too small, so that the interaction between the particle and atoms of the medium cannot be ignored and results in a noticeable Brownian motion of the particle. By the isotropy of the probability distribution of steps of the random walk of state, a displacement of the particle away from $M_{3}^{\sigma}$ is then equally likely. Such a displacement would mean that the particle is now in a superposition of states of different positions. If $\mathbf{a}$ is the initial position and $\mathbf{l}$ is the observed displacement of the particle in $M_{3}^{\sigma}$ during the measurement, then the states $g_{\mathbf{a}, \sigma}$ and $g_{\mathbf{a}+\mathbf{l}, \sigma}$ are distinguishable in the experiment, which means that the superpositions of these states can be observed. We conclude that:

(D) The boundary between quantum and classical occurs for microscopic particles that are sufficiently large in size so that their Brownian motion in the natural medium is noticeable, but not too large, when the Brownian motion trivializes, the state that was initially in the classical space submanifold $M_{3}^{\sigma}$ becomes constrained to $M_{3}^{\sigma}$ and the Schrödinger evolution becomes a Newtonian motion.

Consider now a system consisting of a microscopic particle $P$ and a macroscopic device $D$ capable of measuring the position of $P$. Let say, $D$ is a scintillating screen, small enough to be considered a material point, when treated classically. The particle and the screen form a two-particle system whose state belongs to the product Hilbert space $H=H_{P} \otimes H_{D}$. Since the screen is macroscopic, it interacts with the natural surroundings. The screen also interacts with the particle. By the theorem (A), the motion of state constrained to the submanifold $M_{3}^{\sigma} \otimes M_{3}^{\sigma}$ is Newtonian. Applying the Newtonian physics to the pair we derive that the macroscopic screen $D$ will perform a trivial Brownian motion in the surrounding medium and can be accurately described as being at rest in the lab system, and not affected by the particle $P$. On the other hand, the particle whose position is measured by the screen will perform a random walk in $\mathbb{R}^{3}$ resulting in the normal distribution of the measured position.

We can now make use of the hypothesis (RM) to reconstruct the dynamics of state of the system under the measurement. Suppose that the system is initially in the product state $\Psi=\varphi \otimes g_{\mathbf{a}, \sigma}$, where $\varphi \in H_{P}$ is the state of the particle and $g_{\mathbf{a}, \sigma} \in M_{3}^{\sigma}$ is the state of the screen. Because the Newtonian dynamics of the screen considered as a classical object is not affected by the particle, the hypothesis (RM) can be applied to the screen itself. From (RM), the derived isotropy of the probability distribution of the displacement of state and the established triviality of the motion of state constrained to $M_{3}^{\sigma}$, we conclude that the state of the device will experience a trivial random walk in $C P^{H_{D}}$. In the absence of an external potential, the state can be assumed to be at rest in the space $M_{3}^{\sigma}$. On the other hand, applying (RM) to the measured particle we conclude that the state of the particle interacting with the screen will experience a random walk in the space of states $C P^{H_{P}}$. The state of the system will maintain its product form.

Because the state of the screen was shown to be constrained to $M_{3}^{\sigma}$, the theorem $(\mathbf{A})$ applies and ensures that in the presence of an external potential the screen will continue evolving in 
accord with the Newtonian dynamics. In this case the system remains in a product state with both factors being able to change. In particular, the measured position of the particle can be mechanically recorded with no entanglement between the particle and the screen ever appearing or needing to appear in the process.

The corollary of this is the following result:

(E) Suppose the initial state of the system consisting of a microscopic and a macroscopic particles in the natural surroundings is the product state with the state of the macroscopic particle in the manifold $M_{3}^{\sigma}$. Then, during the interaction of the particles the state of the system remains the product state with the state of the macroscopic particle in $M_{3}^{\sigma}$.

To go back to the issue of consistency of the description of quantum mechanics by macroscopic observers, the answer under the (RM) hypothesis is now clear. The reason for the contradiction in the accounts of Wigner and the friends in the Wigner's friend-type thought experiments is the assumption that a macroscopic observer or the whole lab may exist in a superposition of classical states. Because of the interaction with the surroundings, the evolution of the state of the observers and the lab in the space of states can only happen within the classical space submanifold. The state of the measured system and the observer or the lab remains the product state. This invalidates the no-go theorem in [16] and related results claiming the inconsistency of accounts by the observers. Note that the experiment reported in [17], in which the photons played the role of an observer do not change this conclusion. The photons in the natural surroundings do not evolve by the Hamiltonian represented by a random matrix. The reality as "described" by microscopic particles is different than the one the macroscopic observers are aware of.

Note also that the random matrix description of the interaction between macroscopic bodies and the surroundings allowed us to bypass the decoherence-based approach to the transition from quantum to classical. The modifications of the Schrödinger equation with the goal of accounting for the measurement results and explaining the transition to classicality $[18,19]$ become unnecessary as well. The single outcome of a measurement, the probability of the outcome and the transition from quantum to classical all have now a simple explanation, rooted in the Schrödinger equation, the embedding of the classical space into the space of states and the hypothesis (RM). Let us also remark that the elegance and the scope of the proposed solution to the issue of the relationship between the quantum and classical systems suggests that natural phenomena are happening in the space of states and not in the classical space. If this is the case, the space of states becomes a new arena for all physical processes with the classical space of macroscopic objects as a submanifold of thereof.

\section{Methods}

The isomorphism of the classical space and classical phase space and the submanifolds $M_{3}^{\sigma}$ and $M_{3,3}^{\sigma}$ in the space of states $C P^{L_{2}}$ is explained in [3,4]. The decomposition (1) is obtained by projecting the velocity $\frac{d \varphi}{d t}$ of state under the Schrödinger evolution with an arbitrary Hamiltonian $\widehat{h}=-\frac{\hbar^{2}}{2 m} \Delta+V(\mathbf{x}, t)$ at a point $\varphi=g_{\mathbf{a}, \sigma} e^{i \mathbf{p x} / \hbar}$ in the classical phase space $M_{3,3}^{\sigma}$ onto an orthonormal set of vectors specified by changing the parameters, $\mathbf{a}, \mathbf{p}, \sigma$ that define $\{\varphi\}$ and the phase parameter $\theta$ of a possible constant phase factor $e^{-i \theta}$ of $\varphi$. Calculation of the classical space components of $\frac{d \varphi}{d t}$ at an arbitrary point $\varphi$ in $M_{3,3}^{\sigma}$ yields

$$
\left.\operatorname{Re}\left(\frac{d \varphi}{d t},-\frac{\widehat{\partial \varphi}}{\partial a^{\alpha}}\right)\right|_{t=0}=\frac{p^{\alpha}}{2 m \sigma}
$$


where the hat here and in other calculations denotes normalization. For the momentum space components of $\frac{d \varphi}{d t}$ at $\varphi$ we similarly obtain, assuming that $\sigma$ is small enough to make the linear approximation of $V(\mathbf{x})$ valid:

$$
\left.\operatorname{Re}\left(\frac{d \varphi}{d t}, \frac{\widehat{\partial \varphi}}{\partial p^{\alpha}}\right)\right|_{t=0}=\frac{m w^{\alpha} \sigma}{\hbar}, \text { where } m w^{\alpha}=-\left.\frac{\partial V(\mathbf{x})}{\partial x^{\alpha}}\right|_{\mathbf{x}=\mathbf{a}} .
$$

The components (12) and (13) are tangent to $M_{3,3}^{\sigma}$ and orthogonal to the fibre $\{\varphi\}$. The component of the velocity $\frac{d \varphi}{d t}$ due to change in $\sigma$ (spreading) is orthogonal to the phase space $M_{3,3}^{\sigma}$ and the fibre $\{\varphi\}$, and is equal to

$$
\operatorname{Re}\left(\frac{d \varphi}{d t}, i \frac{\widehat{d \varphi}}{d \sigma}\right)=\frac{\sqrt{2} \hbar}{8 \sigma^{2} m} .
$$

The component of the velocity parallel to the fibre $\{\varphi\}$ is the expected value of energy divided by $\hbar$ :

$$
\operatorname{Re}\left(\frac{d \varphi}{d t},-\frac{\widehat{d \varphi}}{d \theta}\right)=\frac{1}{\hbar}(i \widehat{h} \varphi, i \varphi)=\frac{\bar{E}}{\hbar} .
$$

Calculation of the norm of $\frac{d \varphi}{d t}=\frac{i}{\hbar} \widehat{h} \varphi$ at $t=0$ gives

$$
\left\|\frac{d \varphi}{d t}\right\|^{2}=\frac{\bar{E}^{2}}{\hbar^{2}}+\frac{\mathbf{p}^{2}}{4 m^{2} \sigma^{2}}+\frac{m^{2} \mathbf{w}^{2} \sigma^{2}}{\hbar^{2}}+\frac{\hbar^{2}}{32 \sigma^{4} m^{2}},
$$

which is the sum of squares of the found components. This completes a decomposition of the velocity of state at any point $\varphi$ in $M_{3,3}^{\sigma}$.

The metric relationship (4) follows from the inner product of two states in $M_{3}^{\sigma}$ :

$$
\left(g_{\mathbf{a}, \sigma}, g_{\mathbf{b}, \sigma}\right)=e^{-\frac{(\mathbf{a}-\mathbf{b})^{2}}{8 \sigma^{2}}} .
$$

This expression squared is equal to the right hand side of (4) by the definition of the FubiniStudy distance between states in $C P^{L_{2}}$. The result (5) is obtained in a similar way by evaluating the Fourier transform of a Gaussian function along the way.

The estimate of the diffusion coefficient of a macroscopic spherical particle of radius $1 \mathrm{~mm}$ in still air in normal conditions is based on the Stokes-Einstein relationship

$$
D=\frac{k_{B} T}{6 \pi \eta r}
$$

where $D$ is the diffusion coefficient, $r$ is the radius of the particle, $\eta$ is the dynamic viscosity, $T$ is temperature of the medium and $k_{B}$ is the Boltzmann constant. Using the room temperature and the known value of dynamic viscosity $\eta \sim 10^{-5} \mathrm{~N} \cdot \mathrm{s} / \mathrm{m}^{2}$, we get $D \sim 10^{-12} \mathrm{~m}^{2} / \mathrm{s}$. The variance of the $x$-coordinate of position of the particle is given by $\overline{x^{2}}=2 D t$. If we scatter visible light off the particle to determine its position, the time interval of observation can be estimated to be as short as $10^{-13} \mathrm{~s}$. This amounts to the displacement of the order of $10^{-12} \mathrm{~m}$. The accuracy of measurement is limited by the wavelength $\lambda \sim 10^{-5} \mathrm{~m}$. The Fubini-Study distance between Gaussian states that are $10^{-12} \mathrm{~m}$ apart in $M_{3}^{\sigma}$ with $\sigma \sim 10^{-5} \mathrm{~m}$ is calculated via (4) and is about $10^{-7} \mathrm{rad}$. 


\section{References}

[1] Kryukov, A. On the motion of macroscopic bodies in quantum theory. J. Math. Phys. 58, 082103 (2017).

[2] Kryukov, A. On observation of position in quantum theory. J. Math. Phys. 59052103 (2018).

[3] Kryukov, A. Mathematics of the classical and the quantum. J. Math. Phys. 61082101 (2020).

[4] Kryukov, A. Linear algebra and differential geometry on abstract Hilbert space. Int. J. Math. Math. Sci. 20052241 (2005).

[5] Kryukov, A. Geometric derivation of quantum uncertainty. Phys. Lett. A 370 419-422 (2007).

[6] Ullersma, P. An exactly solvable model for Brownian motion. Physica 32 74-96 (1966).

[7] Zwanzig, R. Nonequilibrium Statistical Mechanics. (Oxford University Press, New York, 2001).

[8] Gaspard, P. Chaos, scattering and Statistical Mechanics. (Cambridge University Press, Cambridge, 1998).

[9] Szasz, D., editor. Hard Ball Systems and the Lorentz Gas. (Springer-Verlag, Berlin, 2000).

[10] Wigner, E. P. On the statistical distribution of the widths and spacings of nuclear resonance levels. Proc. Cambridge Philos. Soc. 47790 (1951).

[11] Bohigas, O., Giannoni, M. J. and Schmit, C. Characterization of Chaotic Quantum Spectra and Universality of Level Fluctuation Laws. Physical Review Letters 521 (1984).

[12] Gaspard, P., Briggs, M. E., Francis, M. K., Sengers, J. V., Gammon, R. W., Dorfman, J. R. and Calabrese, R. V. Experimental evidence for microscopic chaos. Nature 394 865-868 (1998).

[13] Grassberger, P. and Schreiber, T. Microscopic chaos from brownian motion? Nature 401 875-876 (1999).

[14] Cecconi, F., Cencini, M., Falcioni, M. and Vulpiani, A. Brownian motion and diffusion: from stochastic processes to chaos and beyond. Chaos 15(2) 26102 (2005).

[15] Wigner, E. P. Group theory and its application to quantum mechanics of atomic spectra. (Academic Press, New York, 1959).

[16] Frauchiger, D. and Renner, R. Quantum theory cannot consistently describe the use of itself. Nature Communications 93711 (2018).

[17] Proietti, M., Pickston, A., Graffitti, F., Barrow, P., Kundys, D., Branciard, C., Ringbauer, M. and Fedrizzi, A. Experimental test of local observer independence. Science Advances 59832 (2019).

[18] Bassi, A. and Ghirardi, G. Dynamical Reduction Models. Physics Reports 379257 (2003).

[19] Bassi, A., Lochan, K., Satin, S., Singh, T. and Ulbricht, H. Models of wave-function collapse, underlying theories, and experimental tests. Rev. Mod. Phys. 85471 (2013). 